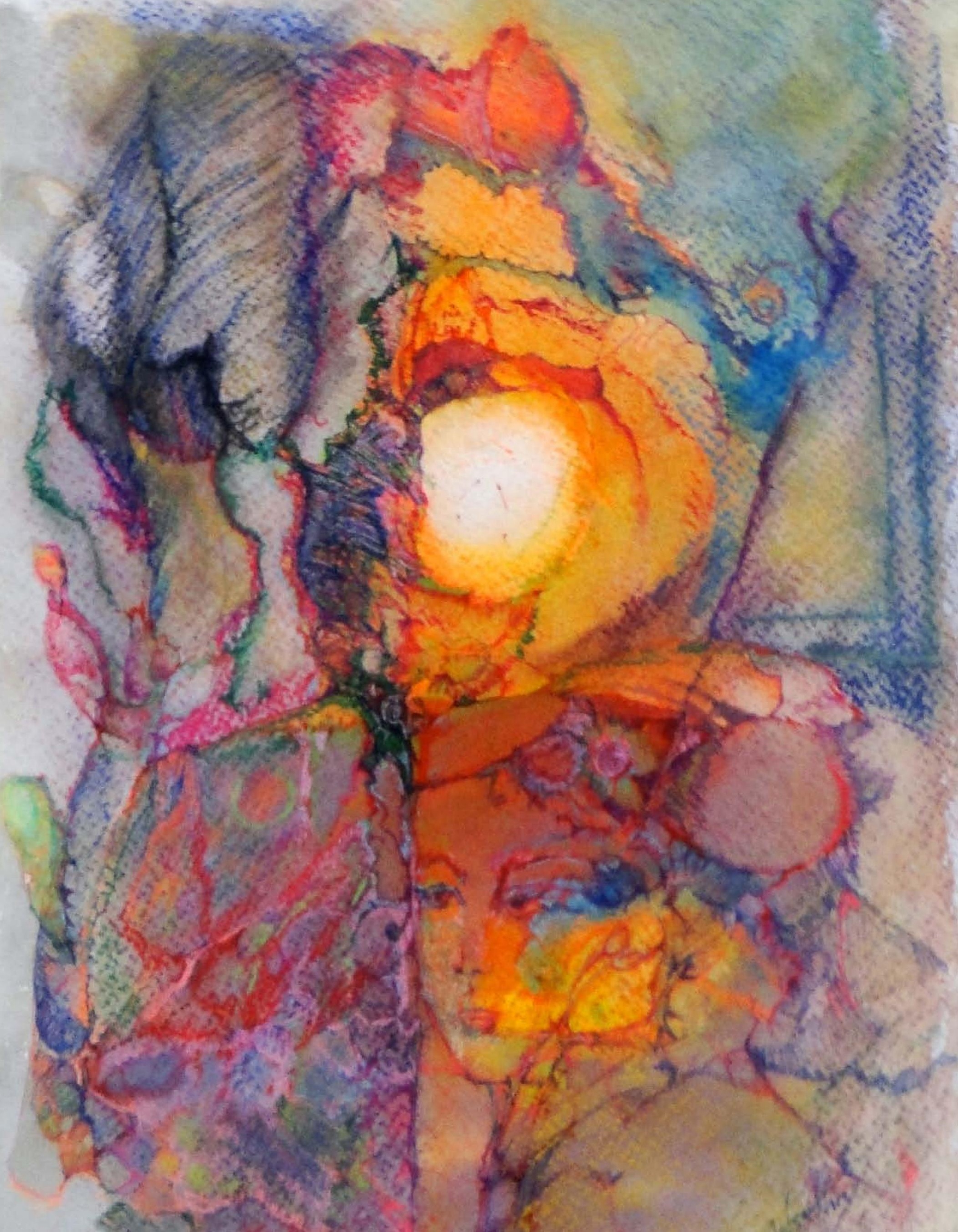




\title{
5 Factores socioculturales que condicionan el embarazo adolescente en los municipios de Intibucá y Jesús de Otoro, departamento de Intibucá
}

\author{
Edith Yolany Zelaya* \\ José Ramón Coto García**
}

\begin{abstract}
RESUMEN. En los últimos años se han presentado estadísticas alarmantes de embarazos adolescentes, en Honduras se estima que el 30\% de los partos ocurren entre jóvenes de 13 a 19 años. En el departamento de Intibucá, de enero a junio del 2010, se han atendido en promedio mensual 77 adolescentes embarazadas entre edades de 10 a 19 años. El embarazo en la adolescencia no sólo representa un problema de salud, también tiene una repercusión en el área social, afectiva y económica. En el caso de los municipios de Intibucá se identifican factores socioculturales que condicionan el embarazo entre las adolescentes entre ellas el machismo, los tabúes alrededor de la educación sexual, el hacinamiento, la falta de espacios de recreación y redes de jóvenes que permitan socializar sus inquietudes y fortalecer la educación sexual.
\end{abstract}

Palabras clave: embarazo, adolescente, partos, educación sexual.

SUMMARY. In recent years there have been alarming statistics of teen pregnancy, it is estimated that in Honduras $30 \%$ of births occur among women in ages from 13 to 19 years. At Intibucá department, from January to June 2010, a monthly average of 77 pregnant teens between the ages of 10-19 years have been attended. The teenage pregnancy is not only a health problem but it also has an impact on the social, emotional and economical areas. In the case of the municipalities of Intibucá socio-cultural factors that influence teenage pregnancy including men chauvinism, taboos about sexual education, overcrowding, lack of recreation and youth networks that allow socializing their concerns and strengthening of sexual education have been identified.

Keywords: pregnancy, teen, births, sexual education.

\section{Introducción}

La maternidad es un rol que se desempeña mejor en edad adulta; cuando ocurre en la adolescencia la mujer presenta dificultades para desempeñar correctamente ese rol, el proceso se perturba y se asumen responsabilidades impropias de esta etapa de la vida.

El embarazo cambia radicalmente la vida de la adolescente y de su familia, además se pueden presentar riesgos altos en la salud de la joven madre y del nuevo ser, sobre todo cuando la madre adolescente pertenece a la población más desprotegida de nuestro país en donde las carencias de necesidades básicas podría ser el factor predisponente número uno. Agregado a esto no deja de ser importante la situación del padre, si éste a su vez es un adolescente, el riesgo es más alto por el apoyo moral y más aun por la carencia de figura paterna que tendrá el nuevo ser.

En los últimos años se han presentado estadísticas alarmantes de embarazo adolecente en Honduras, se estima que el 30\% de los partos ocurre en jóvenes que tienen entre 13 y 19 años de edad; en el hospital Materno Infantil de Tegucigalpa un promedio de 10 adolescentes da a luz cada día.

Recibido: febrero 2011/ aceptado y versión final julio 2011.

* Consultora independiente, Dasonóma, ESNACIFOR, Diplomada en Objetivos de Desarrollo del Milenio con Énfasis en Análisis Socidemográfico.

** Consultor independiente, Lic en Economía, Diplomado en Objetivos de Desarrollo del Milenio con Énfasis en Análisis Socidemográfico. 
Según especialistas la tasa de mortalidad materna en edades de 12 a 14 años ha aumentado en un 300\% en comparación con los demás grupos de edad, muestra que los embarazos son más frecuentes en las edades de 12 a 19 años. En el departamento de Intibucá durante el periodo de enero a junio del 2010, se atendieron en promedio mensual 77 adolescentes embarazadas en edades de 10 a 19 años.

Los embarazos adolescentes generalmente son de alto riesgo, situación que tiene muy alarmadas a las autoridades por el enorme impacto que ello representa para los servicios de salud puesto que requieren atención especializada; aunque en las unidades de salud generalmente existe atención materno infantil, el servicio que prestan es básico, no cuentan con las capacidades para atender partos de alto riesgo, es necesario remitirlos a centros especializados. En este proceso lamentablemente algunos casos no son asistidos a tiempo perdiendo la vida el nuevo ser o su madre.

Los factores que favorecen el crecimiento acelerado de embarazo adolecente son muchos y de diverso tipo: social (precocidad de actividad sexual), familiar (familias desintegradas) y moral (carencia de valores, abusos sexuales), entre otros; por lo que urge una política de Estado, específicamente en el tema de embarazo adolecente, ya que las jóvenes adolescentes no están listas biológica, psicológica, económica e intelectualmente para la procreación.

El propósito de esta investigación es resaltar los factores socioculturales que inciden en el embarazo adolescente y las acciones que instancias como la Secretaría de Salud o autoridades locales realizan para evitarlo.

\section{Metodología}

Se realizó mediante un proceso participativo incluyendo varios actores, como ser la Secretaría de Salud por medio de la Regional de Salud, Hospital Dr. Enrique Aguilar Cerrato, Centros de Salud del municipio de La Esperanza y del municipio de Jesús de Otoro.

El período estudiado revisa documentos del 2007 al 2008 y estadísticas e información actual de enero a junio del 2010.

El proceso de investigación ha considerado las siguientes fases:

- Revisión bibliográfica relacionada con el tema de investigación

- Diseñó y aplicación de instrumentos de recolección de información (encuesta) para adolescentes embarazadas, actores claves o especialistas del tema a quienes se les aplicó el instrumento, gravó sus testimonios para facilitar la sistematización de la investigación, con previa autorización de los mismos.

- Conversatorios con varios actores, principalmente personal voluntario de salud del municipio de Jesús de Otoro, a fin de dar a conocer el objetivo de la investigación, esta fase del proceso investigativo fue muy importante pues los/as participantes en el conversatorio contribuyeron a enriquecer algunos aspectos del trabajo propuesto.

- Revisión de testimonios grabados con expertos para hacer analizar las acciones que realizan y los resultados que obtienen.

- Interpretación y análisis de resultados obtenidos producto de la aplicación de los instrumentos de recolección de información a los/as diferentes actores consultados, para generar explicaciones sobre la problemática del embarazo adolescente y presentar recomendaciones.

\section{El embarazo en adolescentes a nivel nacional}

La adolescencia, es un período de transición, una etapa del ciclo de crecimiento que marca el final de la niñez y anuncia la adultez.

El embarazo en adolescentes es una situación reconocida en el mundo que afecta el bienestar y la salud reproductiva de las mujeres jóvenes, así como el ritmo y la dirección de desarrollo de un país. La maternidad temprana puede entorpecer las perspectivas educativas de una mujer joven, reducir su autonomía social y económica de largo plazo y comprometer tanto su salud como la de su recién nacido. 
En Honduras uno de los países más pobres de América Central, reducir los altos niveles de maternidad adolescente es vital para el mejoramiento de vida de las mujeres y de sus familias, así como de la sociedad en general.

Las estadísticas nacionales sobre embarazo adolescente, son preocupantes según datos de la Organización Mundial de la Salud (OMS) en América Latina, 57 de cada 100 mujeres que dan a luz son adolescentes entre los 12 y 17 años. La Encuesta Nacional de Demografía y Salud (ENDESA) de 2005-2006, reveló que el 22\% de las adolescentes entre los 15 y 19 años estaban embarazadas o tenían un hijo.

Estadísticas oficiales en Centro América demuestran que Honduras tiene la tasa de fecundidad adolescente más alta de la región con 137 nacimientos por cada 1000 mujeres de 15 a 19 años, Nicaragua registra 119, Guatemala 114, El Salvador 104, Belice 95, Panamá 89 y Costa Rica 78. La mayor incidencia en Honduras es en las zonas rurales, donde ocurren hasta 162 nacimientos por cada mil mujeres adolescentes y más entre las jóvenes con poca o ninguna escolaridad.(www.radiolaprimerisima.com)

Según el Instituto de la Juventud de Honduras, actualmente en el país de cada 1000 embarazos 102 son adolescentes, situación que está relacionada con la curiosidad de la primera vez, y un vago conocimiento de la sexualidad reproductiva en los jóvenes y padres de familia. De acuerdo a las autoridades de salud, la cifra de adolescentes embarazadas va en aumento, al grado que en la actualidad se plantea impulsar una campaña de educación intensiva para disminuir los casos de embarazo precoz.

Para la Secretaría de Salud el embarazo en menores se encuentra entre las cinco principales causas de morbilidad en adolescentes. El riesgo de morir por causa de complicaciones en el parto en el caso de adolescentes embarazadas es del 8\%, las complicaciones van desde presión alta, anemia severa, hemorragias, infecciones y bebés de bajo peso (Secretaría de Salud, 2010).

\section{El embarazo en adolescentes en el departa- mento de Intibucá}

La Dirección Regional de Salud No. 10 que comprende el departamento de Intibucá, cuenta con el hospital de área que tiene todo lo necesario para la atención de la maternidad desde su control prenatal hasta el post-parto.

Las estadísticas del 2007 de atenciones de partos de adolescentes en el Hospital Dr. Enrique Aguilar Cerrato ${ }^{1}$ ubicado en la jurisdicción del municipio de Intibucá, registra un 2\% de adolescentes en edades comprendidas entre 10 a 13 años, $27 \%$ en edades entre 14 a 16 años y $71 \%$ en edades entre 17 a 19 años, comportamiento que se repite en el primer trimestre del año 2008 (Estadísticas del HEAC, 2007-2008).

Durante los años 2007 y 2008 de un total 1,005 adolescentes embarazadas que asistieron al hospital Dr. Enrique Aguilar Cerrato, el 33\% procedía del municipio de Intibucá y un 16\% corresponde al municipio de Jesús de Otoro (Estadísticas del HEAC, 2007-2008).

El cuadro No.1 presenta el número de adolescentes según la etapa de adolescencia en que fueron atendidas, el porcentaje más alto se encuentra entre los 17 a 19 años (71.23\%) pero es importante señalar que un $28.77 \%$ lo representan adolescentes menores de 16 años, etapa en la cual el embarazo es de mayor riesgo.

Cuadro 1. Atención de partos según etapas de la adolescencia, atendidos en el Hospital Enrique Aguilar Cerrato en el año 2007 y el primer trimestre del año 2008

\begin{tabular}{|c|c|c|c|c|}
\hline $\begin{array}{c}\text { PARTOS EN ADO- } \\
\text { LESCENCIA SEGÚN } \\
\text { ETAPAS }\end{array}$ & Partos & $\%$ & Partos & $\%$ \\
\cline { 2 - 5 } & 8 & 1.2 & 2 & 4.0 \\
\hline $\begin{array}{c}\text { Temprana (10 a 13 } \\
\text { años) }\end{array}$ & 181 & 27.5 & 50 & 26.0 \\
\hline $\begin{array}{c}\text { Media (14 a 16 } \\
\text { años) }\end{array}$ & 468 & 71.2 & 137 & 73.0 \\
\hline $\begin{array}{c}\text { Tardía (17 a 19 } \\
\text { años) }\end{array}$ & 657 & 100.0 & 189 & 100.0 \\
\hline TOTAL &
\end{tabular}

Fuente: Estadísticas del Hospital Dr. Enrique Aguilar Cerrato, 2008. 
El cuadro No.2 presenta el número de adolescentes que fueron atendidas en la consulta externa del hospital durante enero a junio del 2010, según la procedencia municipal. De los 17 municipios del departamento y de un total de 78 adolescentes el $51.28 \%$ corresponde al municipio de Intibucá, el 14.15\% al municipio de La Esperanza, un $11.54 \%$ al municipio de Jesús de Otoro, seguido de los municipios de San Isidro y Yamaranguila que en conjunto conforman los 5 municipios que reporta el mayor número de casos (Ver cuadro No.2).

Cabe mencionar que estas estadísticas corresponde a las adolescentes que se presentan a su control prenatal en el área de la consulta externa del hospital.

Las estadísticas se ven más dramáticas en el número de adolescentes embarazadas que llegan de emergencia al hospital, según datos presentados, de un total de 462 adolescentes embarazadas 35 están en edades comprendidas entre los 10 y 14 años. El porcentaje más alto según procedencia lo tiene el municipio de

Cuadro No. 2. Procedencia de embarazadas adolescentes atendidas en la consulta externa del Hospital Enrique A. Cerrato en los meses de enero a junio del 2010.

\begin{tabular}{|l|c|c|c|c|}
\multirow{2}{*}{\multicolumn{1}{|c|}{ PROCEDENCIA }} & \multicolumn{2}{|c|}{ Categoría de Edad } & \multirow{2}{*}{ TOTAL } & $\%$ \\
\cline { 2 - 4 } & $10-14$ & $15-19$ & & \\
\hline Intibucá & 6 & 34 & 40 & 51.28 \\
\hline La Esperanza & & 11 & 11 & 14.10 \\
\hline Jesús de Otoro & & 9 & 9 & 11.54 \\
\hline San Isidro & & 6 & 6 & 7.69 \\
\hline Yamaranguila & & 5 & 5 & 6.41 \\
\hline San Fco. de Opalaca & & 2 & 2 & 2.56 \\
\hline Masaguara & & 2 & 2 & 2.56 \\
\hline Dolores & & 1 & 1 & 1.28 \\
\hline Camasca & & & 0 & 0.00 \\
\hline San Juan & & & 0 & 0.00 \\
\hline Colomoncagua & & 1 & 1 & 1.28 \\
\hline Magdalena & & & 0 & 0.00 \\
\hline San Antonio & & & 0 & 0.00 \\
\hline Santa Lucía & 1 & & 1 & 1.28 \\
\hline San Miguelito & & & 0 & 0.00 \\
\hline San Marcos de Sierra & & & 0 & 0.00 \\
\hline Concepción & & & 0 & 0.00 \\
\hline Total & 7 & 71 & 78 & $100 \%$ \\
\hline
\end{tabular}

Fuente: Elaboración propia en base datos estadísticos del Hospital Dr. Enrique Aguilar Cerrato, 2010.
Intibucá con el $34.42 \%$, le sigue Jesús de Otoro con $20.56 \%$ y en tercer lugar Yamaranguila con el $13.20 \%$ superando las cifras registradas en los años 2007 al 2008 (Ver cuadro No. 3).

4.1 Factores socioculturales que inciden en el embarazo de adolescentes en los municipios de Intibucá y Jesús de Otoro.

El embarazo en adolescentes es un fenómeno causado por múltiples factores, principalmente de orden psicosocial, ocurre en todos los estratos sociales sin tener las mismas características en cada uno de ellos.

El comportamiento sexual humano es variable y depende de las normas culturales y sociales especialmente en la adolescencia, pudiendo clasificar a las sociedades humanas de la siguiente manera:

a. Sociedad represiva: en la que se niega la sexualidad, considerando al sexo como un área peligrosa en la

Cuadro No. 3. Número de adolescentes embarazadas según procedencia y categoría de edad que se atendieron de emergencia en el Hospital Enrique A. Cerrato durante enero a junio del 2010

\begin{tabular}{|c|c|c|c|c|}
\hline \multirow[t]{2}{*}{ PROCEDENCIA } & \multicolumn{2}{|c|}{ Categoría de Edad } & \multirow[b]{2}{*}{ Total } & \multirow[b]{2}{*}{$\%$} \\
\hline & $10-14$ & $15-19$ & & \\
\hline Intibucá & 8 & 151 & 159 & 34.42 \\
\hline Jesús de Otoro & 9 & 86 & 95 & 20.56 \\
\hline Yamaranguila & 4 & 57 & 61 & 13.20 \\
\hline Masaguara & 7 & 26 & 33 & 7.14 \\
\hline La Esperanza & 1 & 31 & 32 & 6.93 \\
\hline San Fco. de Opalaca & 4 & 12 & 16 & 3.46 \\
\hline San Miguelito & 1 & 14 & 15 & 3.25 \\
\hline Concepción & & 12 & 12 & 2.60 \\
\hline San Isidro & & 9 & 9 & 1.95 \\
\hline San Juan & & 7 & 7 & 1.52 \\
\hline Dolores & & 6 & 6 & 1.30 \\
\hline San Antonio & 1 & 5 & 6 & 1.30 \\
\hline Camasca & & 6 & 6 & 1.30 \\
\hline San Marcos de Sierra & & 3 & 3 & 0.65 \\
\hline Santa Lucía & & 2 & 2 & 0.43 \\
\hline Colomoncagua & & & 0 & 0.00 \\
\hline Magdalena & & & 0 & 0.00 \\
\hline TOTAL & 35 & 427 & 462 & $100 \%$ \\
\hline
\end{tabular}

Fuente: Propia en base a datos estadísticos del Hospital Dr. Enrique Aguilar Cerrato 2010. 
conducta humana. Considera una virtud la sexualidad inactiva, aceptándola sólo con fines procreativos. Las manifestaciones de la sexualidad pasan a ser fuentes de temor, angustia y culpa, enfatizando y fomentando la castidad prematrimonial.

b. Sociedad restrictiva: tiene tendencia a limitar la sexualidad, separando tempranamente a los niños y niñas por su sexo. Se aconseja la castidad prematrimonial, otorgando al varón cierta libertad. Presenta ambivalencia respecto al sexo, siendo la más común de las sociedades en el mundo.

c. Sociedad permisiva: tolera ampliamente la sexualidad, con algunas prohibiciones formales (la homosexualidad). Permite las relaciones sexuales entre adolescentes y el sexo prematrimonial. Es un tipo social común en países desarrollados.

d. Sociedad alentadora: para la cual el sexo es importante y vital para la felicidad, considerando que el inicio precoz del desarrollo de la sexualidad favorece una sana maduración del individuo. La pubertad es celebrada con rituales religiosos y con instrucción sexual formal. La insatisfacción sexual no se tolera y hasta es causal de separación de pareja. Son sociedades frecuentes en África ecuatorial, la Polinesia y algunas islas del Pacífico.

4.2 Factores socioculturales que determina un embarazo en adolescentes desde el punto de vista de profesionales especialistas en el tema

- Menarquía temprana (Primera menstruación): se inicia la maduración sexual, predomina la coquetería del amor platónico, el enamoramiento y comienzan a perder interés en los padres. Años atrás la menarquía se presentaba entre los 13 y 15 años pero actualmente se presenta entre los 9 y 10 años.

- Inicio precoz de relaciones sexuales: la curiosidad y fantasía de la primera vez, y no existe la madurez emocional necesaria para implementar una adecuada prevención.

- Falta de educación sexual y reproductiva: primero hay "tabúes" que deben ser eliminados para poder explicar o aconsejar a los y las adolescentes sobre la sexualidad. En la jefatura de enfermería del hospital opinan que tienen que ver los centros educativos ya que al hablar de sexualidad: "el abordaje el tema sobre educación sexual es muy superficial, no se le da a los y las jóvenes muchos elementos para prevenir los embarazos, no es lo mismo que los explique un especialista."

- Patrones de machismo a nivel de la familia: la repetición de patrones y culturas donde prevalece el machismo, reduce las oportunidades de estudio de las adolescentes y se ve como normal que a temprana edad se conviertan en madres. Culturalmente se cree que los padres son dueños de los hijos coartando sus iniciativas induciéndoles a hacer los que ellos desean y no lo que aspiran los hijos.

El machismo unido al alcoholismo es otro factor determinante para poner en riesgo a una adolescente.

"la culpa es de mi hija, es que ella se baña y se cambia en frente de mí y yo ya bolo solo actuó",

Testimonio de un abusador.

Es claro que si una adolescente vive en una familia con problemas de alcoholismo corre el riesgo de ser abusada y por ende salir embarazada.

- Familias viviendo en hacinamiento: en las comunidades rurales es común que más de una familia viva en la misma casa sin tener las condiciones necesarias para dividir las habitaciones y evitar que los niños, niñas y adolescentes sean testigos de las relaciones sexuales de los mayores. Según la Fiscalía de la Niñez de Intibucá: "la mayoría de los casos de padres que violan a sus hijas son actos que las madres los conocen pero no los denuncian". El encargado de Epidemiologia en Intibucá enuncia que el hacinamiento en el área rural provoca que los niños sean testigos del trato que el padre le da a la madre para tener relaciones sexuales y ellos comienzan a cortejar a sus primas o vecinas confundiendo el cortejo con el abuso sexual, así surgen los incestos, el abandono de hogar de las niñas o adolescentes que para no seguir en el abuso forman otro hogar a una edad de 15 a 16 años. 
- Familia disfuncional: uniparentales o de conductas promiscuas, que ponen de manifiesto la necesidad de protección de una familia consciente, con buen dialogo padres-hijos. Su ausencia genera carencias afectivas que la joven no sabe resolver, impulsándola a relaciones sexuales que tienen un carácter de sometimiento.

- Migraciones recientes: provoca pérdida del vinculo familiar. Ocurre con el traslado de las jóvenes a las ciudades en busca de trabajo y aún con motivo de estudios superiores. Así mismo la migración de sus padres a otros países dejando a los adolescentes solos o con encargados que no pueden orientarlos.

- La influencia desorientada de la televisión y el internet: adolescentes con pensamientos mágicos o fantasías de telenovelas juveniles y la pornografía contribuye con actitudes de ser independientes e impulsivos, de lanzarse a una vida social de fiesta y diversión sin el control necesario de uno de sus padres. La televisión y el internet se han convertido en las fuentes de información más accesibles para los adolescentes y sin supervisión de un adulto son impulsados a poner en práctica lo que ven sin medir consecuencias.

- Falta de espacios recreativos para los jóvenes: los adolescentes no tienen espacios recreativos en los cuales pueden hacer un uso correcto de su tiempo libre, además en nuestras comunidades no se crean espacios donde los y las jóvenes pueden interactuar sobre temas que les concierne o aprender deportes, oficios o manualidades que les llame la atención y les sirva de pasatiempo.

- Bajo nivel educativo: marcado por un desinterés general. Cuando hay un proyecto de vida que prioriza alcanzar un determinado nivel educativo y posponer la maternidad para la edad adulta es más probable que la joven aún teniendo relaciones sexuales, adopte una prevención efectiva del embarazo.

- Controversias entre su sistema de valores y el de sus padres: cuando en la familia hay una severa censura hacia las relaciones sexuales entre adolescentes, muchas veces los jóvenes inician su actividad sexual por rebeldía sin considerar las consecuencias.

\section{Estudios de caso}

Para efectos de la investigación se realizaron entrevistas a adolescentes embarazadas, consultándoles datos generales como su edad, estado civil, y su actual situación de embarazo.

El cuadro No. 4 presenta el resultado de las encuestas realizadas a 9 adolescentes embarazadas. El tema y la situación misma pone a las adolescentes esquivas y no muy fácilmente brindan información personal.

Basándonos en los resultados de las adolescentes encuestadas se concluyen las siguientes consideraciones:

- El estado civil en su mayoría es el de madres solteras. Las que están en unión libre dejan de estudiar y las solteras que presentan mejores condiciones de vida pueden tener el apoyo de sus familiares (de 9 sólo 3 estudian). En otros casos aunque sus padres les apoyan probablemente el ingreso familiar es poco para ofrecer a la adolescente el estudio si la prioridad es su embarazo y la preparación para el parto.

- Las condiciones de vivienda son precarias, la mayoría cuenta con servicios básicos como agua, luz, sin embargo, viven en hacinamiento, los casos extremos con una vivienda alquilada sin servicios básicos.

- Al momento de consultarles iPor qué salió embarazada?, de las 9, 3 contestaron que "no sabía" y las 5 restantes contestaron "por descuido"; a esto le agregamos que información recibieron para prevenir y sus respuestas fueron: "poca o ninguna" en el caso de las de nivel de primaria; las de nivel de secundaria responden: "en el colegio nos hablan de anticonceptivos".

- Se les consultó sobre la reacción al saber que estaban embarazadas las respuestas fueron: "me fui de la casa", "miedo por la reacciones de mi familia", "me asuste no sabía qué hacer, quería morirme", "triste, tuve que ir a vivir con el papá del bebe".

- También se les consultó sobre los cambios experimentados y se encontraron situaciones como: "ya no salgo, me da pena", "ya no es lo mismo mis papás no me hablan", "no puedo trabajar", "mayor obligación y no me dejan salir", "tengo menos amigos", "sólo paso en la casa, no puedo trabajar". 
Cuadro No. 4. Resultados condensados de encuestas a adolescentes embarazadas en los municipios de Jesús de Otoro e Intibucá

\begin{tabular}{|c|c|c|c|c|c|c|c|c|c|}
\hline No. & Edad & $\begin{array}{l}\text { Estado } \\
\text { civil }\end{array}$ & $\begin{array}{l}\text { Estudia } \\
\text { Si/No }\end{array}$ & $\begin{array}{l}\text { Grado de } \\
\text { Escolaridad }\end{array}$ & $\begin{array}{l}\text { Edad inicio } \\
\text { de actividad } \\
\text { sexual }\end{array}$ & Condiciones de vivienda & Con quien vive & $\begin{array}{c}\text { Tiene } \\
\text { control } \\
\text { prenatal }\end{array}$ & Procedencia \\
\hline 1 & 14 & Soltera & No & $6^{\text {to }}$ grado & 13 & $\begin{array}{l}\text { Bahareque, sin agua ni } \\
\text { letrina, una pieza }\end{array}$ & 1 cuñada & $\mathrm{Si}$ & $\begin{array}{l}\text { Jesús de } \\
\text { Otoro }\end{array}$ \\
\hline 2 & 16 & Unión libre & No & $3^{\text {ro }}$ Ciclo & 16 & $\begin{array}{l}\text { Tiene servicios básicos, } 3 \\
\text { piezas }\end{array}$ & Con el marido & $\mathrm{Si}$ & Intibucá \\
\hline 3 & 14 & Soltera & $\mathrm{Si}$ & $2^{\text {do }}$ Ciclo & 14 & $\begin{array}{l}\text { Tiene servicios básicos, } 3 \\
\text { piezas }\end{array}$ & $\begin{array}{l}4 \text { (mamá, abuela y } 2 \\
\text { hermanas. }\end{array}$ & $\mathrm{Si}$ & $\begin{array}{l}\text { Jesús de } \\
\text { Otoro }\end{array}$ \\
\hline 4 & 17 & Soltera & No & $5^{\text {to }}$ grado & 15 & $\begin{array}{l}\text { Sin luz, piso de tierra, } \\
2 \text { habitaciones para } 6 \\
\text { personas. }\end{array}$ & Padres y hermanos & $\mathrm{Si}$ & $\begin{array}{l}\text { Jesús de } \\
\text { Otoro }\end{array}$ \\
\hline 5 & 15 & Soltera & No & $6^{\text {to }}$ grado & 14 & $\begin{array}{l}\text { Piso de tierra, } 2 \text { piezas para } \\
5 \text { personas }\end{array}$ & Padres y hermanos & $\mathrm{Si}$ & Intibucá \\
\hline 6 & 16 & Soltera & $\mathrm{Si}$ & $2^{\text {do }}$ Ciclo & 16 & $\begin{array}{l}\text { Tiene servicios básicos, } 3 \\
\text { piezas }\end{array}$ & Suegros & No & $\begin{array}{l}\text { Jesús de } \\
\text { Otoro }\end{array}$ \\
\hline 7 & 18 & Unión Libre & No & $5^{\text {to }}$ grado & 16 & $\begin{array}{l}\text { Casa alquilada, tiene piezas } \\
\text { separadas }\end{array}$ & $\begin{array}{l}\text { Marido, padres y } \\
\text { hermana }\end{array}$ & $\mathrm{Si}$ & $\begin{array}{l}\text { Jesús de } \\
\text { Otoro }\end{array}$ \\
\hline 8 & 19 & Unión libre & No & $3^{\text {ro }}$ Ciclo & 19 & Tiene servicios básicas & $\begin{array}{l}\text { Con familia del } \\
\text { marido }\end{array}$ & $\mathrm{Si}$ & $\begin{array}{l}\text { Jesús de } \\
\text { Otoro }\end{array}$ \\
\hline 9 & 16 & Soltera & Si & $1^{\text {ro }}$ Bach & 15 & 2 piezas para 3 personas & Con abuelos. & & Intibucá \\
\hline
\end{tabular}

Fuente: Elaboración propia según entrevistas realizadas con adolescentes.

\section{Acciones o estrategias que se ejecutan para prevenir el embarazo en adolescentes en la Región Sanitaria No. 10}

En lo que corresponde a la Región de Salud No. 10 que pertenece al departamento de Intibucá las acciones que se están implementando son las siguientes:

- Estrategia RAMNI: pretende atender a nivel familiar la salud del individuo, su familia y su alrededor abordando temas sobre la sexualidad reproductiva, planificación familiar, enfermedades de transmisión sexual, la atención de población vulnerable: niños, adultos mayores etc. La estrategia incluye los anuncios radiales sobre cómo prevenir enfermedades y embarazos.

- Implementación de nuevo formato de atención a adolescentes: se están conformando dos proyectos piloto de clínicas de atención a adolescentes en la cuales además de la evaluación meramente física también se evaluará la psiquis de los y las jóvenes, para prevenir problemas de drogadicción y suicidio. Actualmente se cuenta con el edificio para la clínica de labor y parto en el municipio de Jesús de Otoro, se está gestionando el equipamiento para lograr su inauguración a final del año 2010. El propósito principal de la clínica será mejorar el acceso a la atención primaria en enfermedades comunes y principalmente a la de labor y parto disminuyendo la demanda de atención del Hospital Enrique Aguilar Cerrato.

A nivel de capacitación se implementaran charlas de sexualidad reproductiva en centros educativos desde $4^{\text {to }}$ a $5^{\text {to }}$ grado debido a que es la etapa en la que se está presentando la primera menstruación.

- Orientaciones en los centros de salud: no es muy frecuente la visita de adolescentes a los centros de salud, sin embargo, el personal médico ya está consciente de que deben brindar orientación a las adolescentes que llegan embarazadas aconsejándolas de que eviten en corto tiempo un segundo embarazo. La labor de prevención que realizan los centros de salud es a través de las capacitaciones con los voluntarios de salud quienes se convierten en los mensajeros dentro de las comunidades, también se 
exponen murales en los pasillos o salas de espera, emplean anuncios radiales y acuden al llamado de centros educativos cuando les solicitan charlas sobre educación sexual.

- Nueva política de género que implementará Plan en Honduras: esta ONG, está revisando una nueva política de género que incluya como programa la educación sexual y reproductiva con jóvenes y sus padres.

- Alianzas estratégicas de salud con ONG's: la Región de Salud No. 10 continuamente está promoviendo

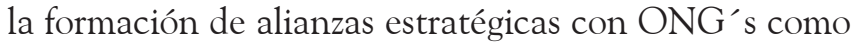
Visión Mundial, Save The Children, Plan en Honduras, el propósito fundamental es que juntos puedan afrontar los problemas de salud en la población vulnerable del departamento.

\section{Conclusiones y recomendaciones}

\subsection{Conclusiones}

- La educación en salud sexual y reproductiva impartida en los centros educativos de estos municipios no contribuye con la prevención del embarazo adolescente, es una asignatura pendiente, el abordaje de los temas sexuales, se hace mediado por una serie de "tabúes", que limitan un ambiente de confianza entre el alumno y maestro(a) complicándose aún más cuando es abordado entre padres e hijos.

- Los resultados de este estudio confirman que en el área rural es común que las familias vivan en hacinamiento, esta necesidad básica insatisfecha favorece las relaciones sexuales a temprana edad, especialmente por la cultura machista y de sometimiento en que viven las mujeres, contribuyendo a que se produzcan casos incestuosos.

- El uso sin supervisión de la tecnología de comunicación e información es actualmente una práctica cotidiana de la juventud adolescente, especialmente la visita a páginas pornográficas en las cuales se promociona el sexo de manera abierta y sin restricciones.

\subsection{Recomendaciones}

A partir de las entrevistas y conversatorios realizados se presentan las recomendaciones sugeridas.

- Implementar un programa de atención en salud sexual y reproductiva ad-hoc dirigido las adolescentes embarazadas, que contemple las particularidades propias de su edad que adopte un abordaje preventivo.

- Implementar en las comunidades rurales un programa de capacitación en salud sexual y reproductiva dirigida a los(as) adolescentes y sus padres. La capacitación puede ser impartida por los/as guardianes de salud y las parteras con la supervisión del personal del hospital.

- La enseñanza de la salud sexual y reproductiva en los centros educativos en todos los niveles debe ser abordada con objetividad, promoviendo en los/as maestros(as) la eliminación de tabúes y prejuicios e impulsando el rescate de valores dentro de las familias.

- Organizar un programa de sensibilización/atención/ capacitación dirigido a padres y madres de familia que tienen en su seno hijos e hijas adolescentes conflictivos o que están en riesgo con el propósito de mejorar las relaciones familiares haciendo énfasis en la comunicación entre padres e hijos, en la comprensión del comportamiento adolescente y así evitar comportamientos extremos, especialmente los suicidios.

- Es importante promover un programa de oportunidades juveniles que haga énfasis en el uso del tiempo libre en aspectos útiles, con recreación sana, participación comunitaria: voluntariados, redes juveniles con propósitos sociales encaminados al desarrollo de la comunidad en que viven. 


\section{Bibliografía}

- Álvarez Rodríguez, Bertha Elizabeth, 2006, Incidencia e impacto del embarazo adolecente entre los pobres

- Castillo, Beatriz,2010, Fadep News, embarazos de adolecentes en Honduras

- Hospital Jesús Enrique Aguilar Cerrato, Departamento de Estadísticas, 2007-2010.

- http://www.elheraldo.hn

- Molero, Janire (2010) El Mundo es. Bebés virtuales para prevenir el embarazo adolescente en Honduras.
- Pantelides, Edith A(2009) Aspectos sociales en el embarazo y la fecundidad adolescente en América Latina.

- Prensa latina, febrero del 2008, Tegucigalpa.

- Reyes Turcios, Nidia R (2006) Factores que predisponen la ocurrencia de embarazos en adolecentes en colonia Flor del Campo Comayagüela, Honduras.

- Zavala Andino, Virginia (2008) Embarazo en adolescentes en el departamento de Intibucá.

- www.radiolaprimerisima.com 